\begin{tabular}{|c|c|}
\hline Title & Novel Plasmon-Resonant Terahertz-W ave Emitter U sing a Double- Decked HEMT Structure \\
\hline Author(s) & Suemitsu, T.; Meziani, Y. M.; Hosono, Y .; Hanabe, M.; Otsuji, T.; Sano, E. \\
\hline Citation & $\begin{array}{l}\text { Device Research Conference, } 2007 \text { 65th, 157-158 } \\
\text { https://doi.org/10.1109/DRC.2007.4373696 }\end{array}$ \\
\hline Issue Date & 2007 \\
\hline Doc URL & http:/hdl.handle.net/2115/28784 \\
\hline Rights & $\begin{array}{l}\text { O2007 IEEE. Personal use of this material is permitted. However, permission to reprint/republish this material for } \\
\text { advertising or promotional purposes or for creating new collective works for resale or redistribution to servers or lists, } \\
\text { or to reuse any copyrighted component of this work in other works must be obtained from the IEEE. }\end{array}$ \\
\hline Type & proceedings \\
\hline Note & 65th Device Research Conference, The University of Notre Dame, South Bend, Indiana, June18-20, 2007 \\
\hline File Information & DRC2007.pdf \\
\hline
\end{tabular}

Instructions for use 
June 18 - 20, 2007

The University of Notre Dame

South Bend, Indiana

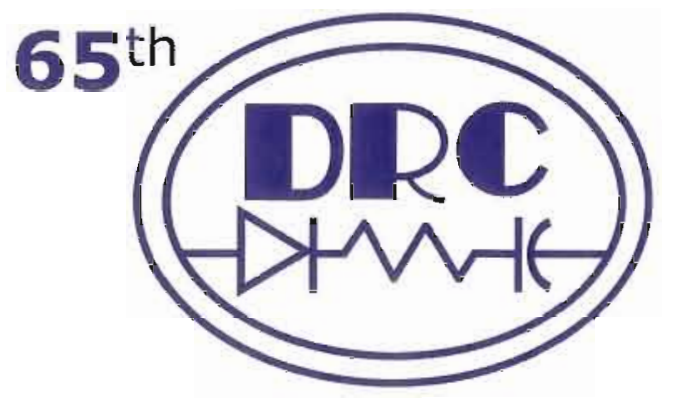

\section{DEVICE}

- IEEE

RESEARCH

CONFERENCE

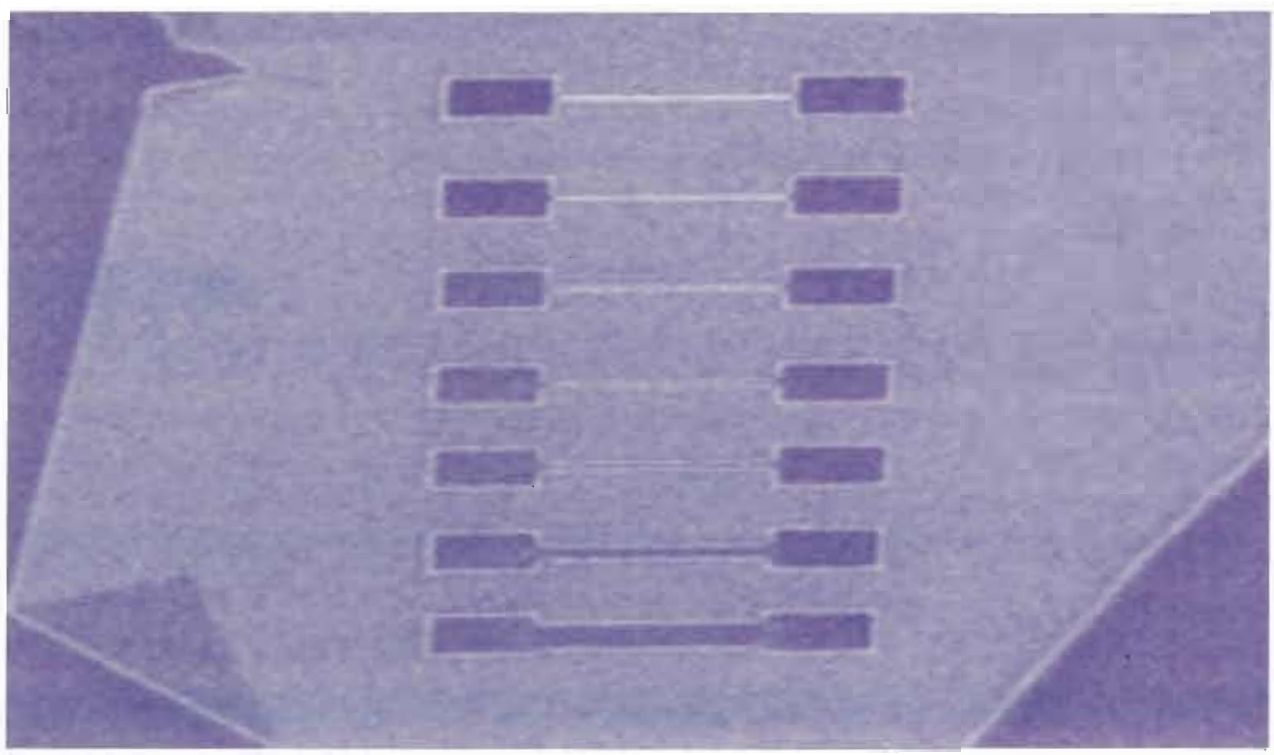

Conference Digest Otsuji 


\title{
Novel Plasmon-Resonant Terahertz-Wave Emitter Using a Double-Decked HEMT Structure
}

\author{
T. Suemitsu, Y. M. Meziani, Y. Hosono, M. Hanabe, T. Otsuji, and E. Sano ${ }^{\dagger}$ \\ Research Institute of Electrical Communication, Tohoku University, 2-1-1 Katahira, Aoba, Sendai 980-8577, Japan \\ †) Research Center for Integrated Quantum Electronics, Hokkaido University, N13 W8, Sapporo 060-8628, Japan \\ Phone:+81-22-217-6106, e-mail: sue@riec.tohoku.ac.jp
}

A new plasmon-resonant THz-wave emitter is fabricated and characterized. The heterostructure of the device consists of double-decked high electron mobility transistor (HEMT) and the upper-deck HEMT works as a grating antenna to convert the non-radiative plasmonic wave in the lower-deck HEMT channel to radiative THz electromagnetic wave. This conversion can be done more efficiently than a metal grating antenna. The experimental observed clear evidence of the THz-wave emission from the double-decked HEMT device.

Exploring the $\mathrm{THz}$ frequency range has been drawing increasing attention to realize compact, light-weight, solidstate $\mathrm{THz}$ devices for security, sensing, and communication. Two-dimensional (2D) plasma oscillation in semiconductors has been studied as a way to generate, detect, and multiply electromagnetic waves in the THz frequency range [1,2]. In our previous work, the grating dual-gate HEMT structure was employed to produce the 2D plasmon [3]: By applying appropriate biases to the dual gate, 2D electron gas (2DEG) is periodically localized in the channel layer which works as the $2 \mathrm{D}$ plasmon cavity to enhance the plasmon instability, resulting in the observation of THz-wave emission at room temperature. In this work, in order to produce the periodically-localized 2DEG, the double-decked HEMT structure is employed. The upper deck channel is then periodically etched as shown in Fig. 1 to form the uncapped region where the 2DEG concentration becomes lower than the capped region without any external gate bias. The HEMT structure consists of the InGaP/InGaAs/GaAs heterostructure with a selective doping in the InGaP layer. For the source/drain ohmic contacts, $\mathrm{AuGe} / \mathrm{Ni}$ was lifted off and annealed after the upper-deck HEMT was selectively etched. The intrinsic device area has a geometry of 30x75 $\mu \mathrm{m}$, where the grating pattern is replicated on the upper-deck HEMT layer. The grating consists of 80 -nm lines and 350-nm lines aligned alternately with a spacing of $100 \mathrm{~nm}$ (Fig. 2).

The plasmon itself is non-radiative. Hence, in order to emit THz wave, an antenna is necessary to transform this longitudinal wave to a radiative transverse wave. The grating gate in the previous work does work as an antenna. However, theoretical study revealed that a low-conductive gate electrode, in which the electron concentration should be comparable to that of 2DEG channel, is preferred in the THz frequency range to enhance the efficiency of field emission [4]. In this case the metal gate has a disadvantage that there are much more electrons in it than the semiconductor channel. In the double-decked HEMT structure in this work, on the other hand, the upper deck channel serves as the grating antenna and its structure is exactly the same as the lower channel. Therefore more intensity in the emitted THz wave is expected.

The experimental setup is shown in Fig. 3. The device is illuminated by a $1.5-\mu \mathrm{m} \mathrm{CW}$ laser from the backside. Electromagnetic wave emitted from the device is detected with a Si bolometer cooled down to $4.2 \mathrm{~K}$ through a filter permitting a frequency range between 0.5 to $3 \mathrm{THz}$. A lock-in technique is used for the measurement. The output of the bolometer is shown in Fig. 4 as a function of the drain bias voltage $\left(\mathrm{V}_{\mathrm{ds}}\right)$. The $\mathrm{V}_{\mathrm{ds}}$ increases to the knee voltage from which the transistor is operated in the saturation region. The bolometer signal starts increasing at around $6 \mathrm{~V}$ and two clear peaks are observed at 8 and $11 \mathrm{~V}$. These features were observed with good reproducibility as shown in Fig. 4. For comparison, Fig. 5 shows the same measurement carried out on the device with the metal grating dual-gate HEMT structure device used in the previous work that confirmed the THz-wave emission by the photo response and electro-optic sampling measurements [3]. Note that the $\mathrm{V}_{\mathrm{ds}}$ range is different from those of Fig. 4 because the double-decked HEMTs in this work suffer from large parasitic source and drain resistance. Nevertheless the double-decked device exhibits more drastic change in the bolometer signal with increasing $\mathrm{V}_{\mathrm{ds}}$. This result supports the idea of low-conductive gate stack to enhance the THz radiation efficiency [4], and therefore indicates that the proposed double-decked HEMT structure is a promising candidate to realize solid-state THz-wave emitters with high power and large efficiency.

In conclusion, strong THz-wave emission is observed from the newly proposed plasmon-resonant THz-wave emitter with a double-decked HEMT structure. Further improvement in the intensity and efficiency of the THz emission will be expected by optimizing device parameters.

This work was partially funded by the Ministry of Internal Affairs and Communications and the Japan Society of the Promotion of Science through the programs of SCOPE and Grant-in-Aid for Scientific Research (Category S), respectively. A part of this work has been carried out at the Laboratory for Nanoelectronics and Spintronics, Research Institute of Electrical Communication in Tohoku University.

1. M. Dyakonov and M. Shur, Phys. Rev. Lett., vol. 71, pp. 2465-2468, 1993.

2. V. Ryzhii and M. Shur, Jpn. J. Appl. Phys., vol. 41, pp. L922-L924, 2002.

3. T. Otsuji, Y. M. Meziani, M. Hanabe, T. Ishibashi, T. Uno, E. Sano, Appl. Phys. Lett., vol. 89, pp. $263502,2006$.

4. T. Otsuji, M. Hanabe, T. Nishimura, E. Sano, Optics Express, vol. 14, pp. 4815-4825, 2006. 


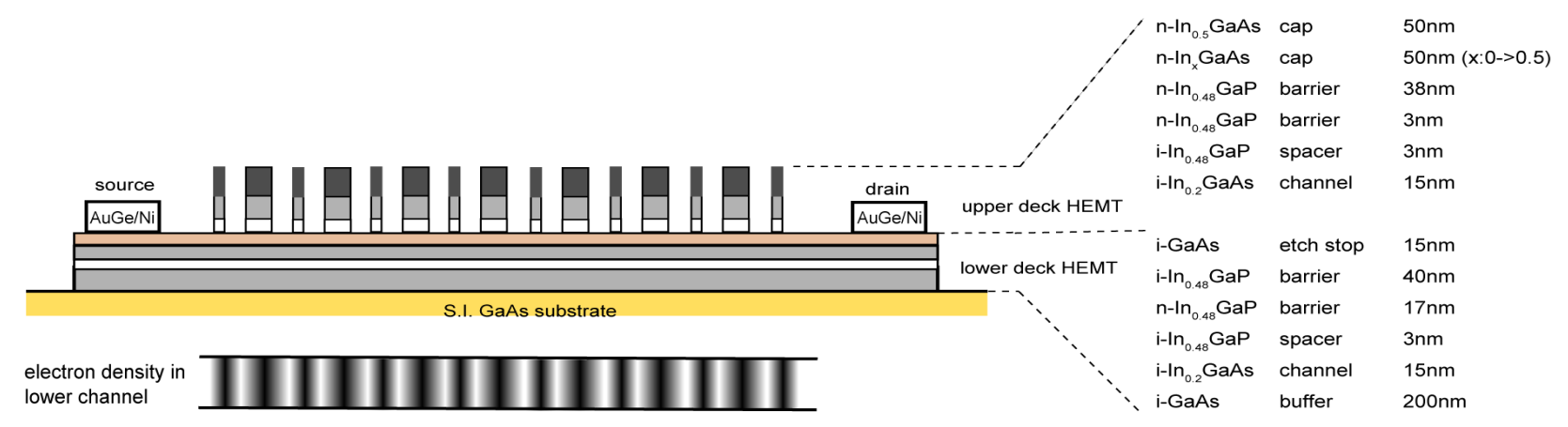

Fig. 1: Cross sectional view of novel plasmon-resonant THz-wave emitter with double-decked HEMT structure. 2D plasmon is generated in the lower-deck HEMT channel and the upper-deck HEMT works as a grating antenna.

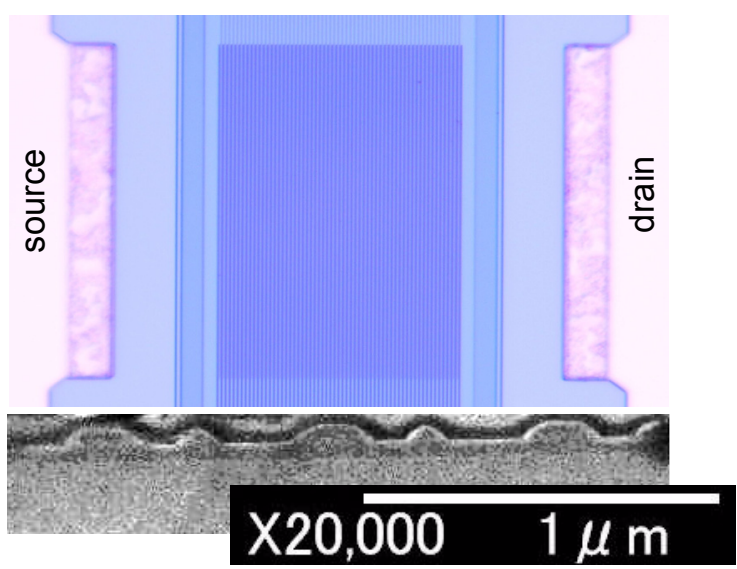

Fig. 2: Top view microphotograph of fabricated doubledecked HEMT plasmon-resonant THz-wave emitter (top) and cross sectional scanning electron microscope image of grating upper-deck HEMT (bottom).

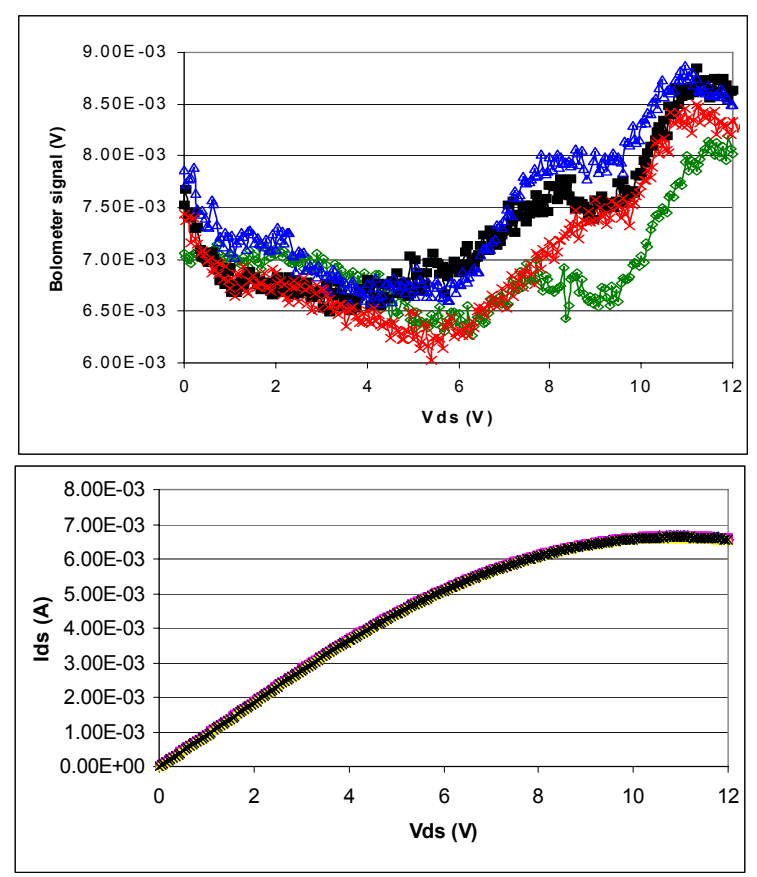

Fig. 4: Bolometer output (top) and drain current $\left(\mathrm{I}_{\mathrm{ds}}\right)$ (bottom) of double-decked HEMT THz-wave emitter as a function of drain voltage $\left(\mathrm{V}_{\mathrm{ds}}\right)$. Measurement took place four times. Bolometer signal increases rapidly at $\mathrm{V}_{\mathrm{ds}}>6 \mathrm{~V}$, which is a clear evidence of the THz-wave emission.

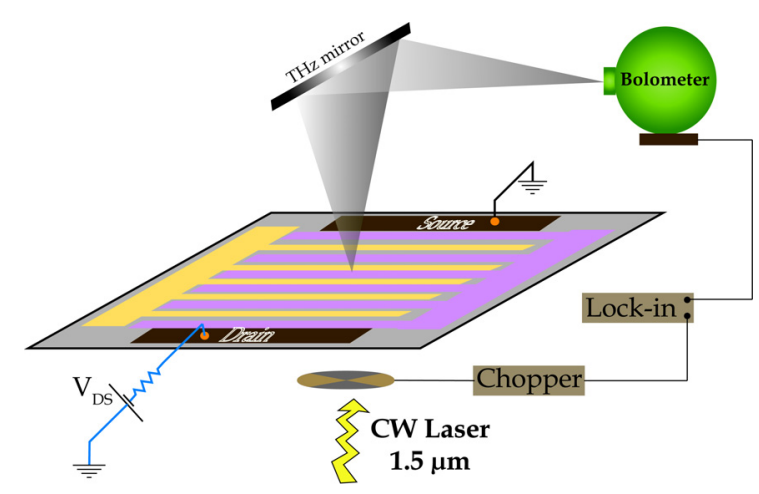

Fig. 3: Experimental setup. $1.5-\mu \mathrm{m} \mathrm{CW}$ laser, chopped for lock-in measurement, illuminates the device from backside. Radiated electromagnetic wave is detected with Si bolometer sensing a frequency range between 0.5 to $3 \mathrm{THz}$. Measured at room temperature.

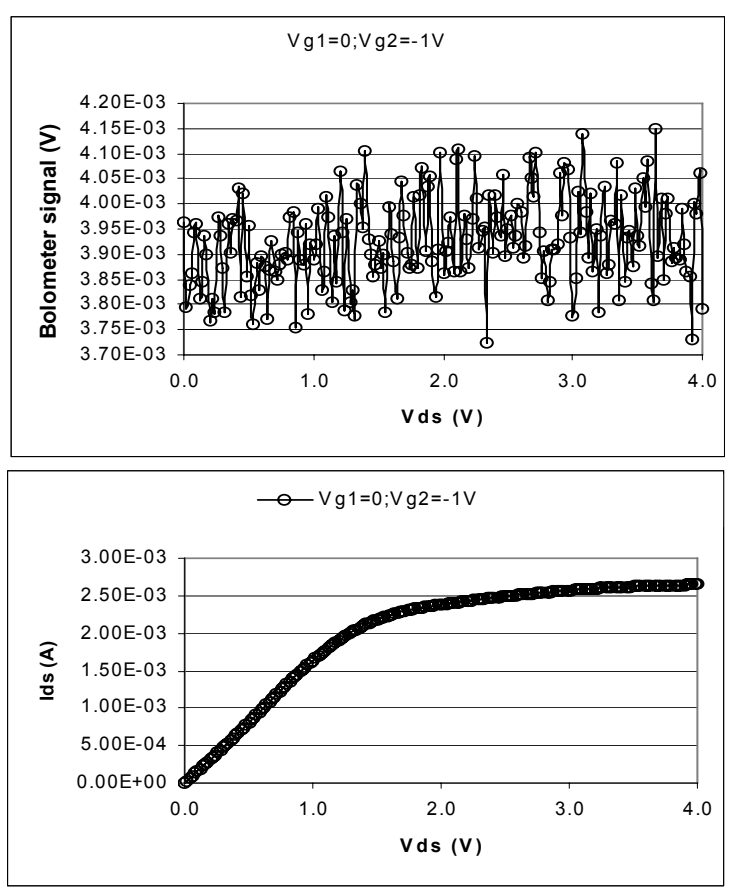

Fig. 5: Same measurement for metal grating dual-gate THz-wave emitter reported previously [3]. The gate biases $\left(\mathrm{V}_{\mathrm{g} 1}=0 \mathrm{~V}\right.$ and $\left.\mathrm{V}_{\mathrm{g} 2}=-1 \mathrm{~V}\right)$ set at point that the highest emission signal was observed. Although $\mathrm{THz}$ emission was confirmed also on this device, the bolometer signal is much weaker than that shown in Fig. 4. 